\title{
Estilos de aprendizaje y evaluación formativa: Estudio con universitarios de Educación Física chilenos
}

\section{Learning styles and formative assessment: Study with Chilean Physical Education university students}

\author{
Jaime Serra-Olivares*, Javier García-Rubio ${ }^{23}$, Pedro Gil Madrona ${ }^{4}$, Consuelo Cejudo Armero ${ }^{4}$ y Sixto González Víllora \\ 1 Pedagogia en Educación Física. Facultad de Educación. Universidad Católica de Temuco. \\ 2 Facultad de Educación. Universidad Autónoma de Chile. \\ 3 Facultad de Ciencias del Deporte. Universidad de Extremadura. \\ 4 Departamento de Didáctica de la Expresión Musical, Plástica y Corporal. Facultad de Educación de Albacete. UCLM. \\ 5 Departamento de Didáctica de la Expresión Musical, Plástica y Corporal. Facultad de Educación de Cuenca. UCLM.
}

\begin{abstract}
Resumen: La evaluación contribuye a la formación del aprendizaje, facilitando el avance del estudiante. La evaluación formativa se presenta como una estrategia eficaz dentro de la formación inicial docente. Se analizaron las percepciones sobre la evaluación de universitarios de Educación Física chilenos $(n=95)$, en función de su estilo de aprendizaje, género y curso académico. Se utilizaron el Cuestionario de Estilos de Aprendizaje y la versión en castellano del Assessment Experience Questionnaire. Los resultados mostraron percepciones negativas sobre el proceso evaluativo. Se observaron diferencias significativas en las percepciones sobre el uso del feedback en función del estilo de aprendizaje $(F=1.062, p=.004)$. Se observaron diferencias significativas en la percepción de la cantidad de esfuerzo $(F=5.888$, $p=.000)$, la adecuación de la evaluación $(F=3.282, p=.015)$ y la aproximación superficial al aprendizaje $(F=5.472, p=.001)$, en función del curso académico. El género no fue determinante de las percepciones. Los hallazgos sugieren la utilización de procedimientos de evaluación tradicional con los encuestados. Se encontraron relaciones entre el estilo de enseńanza y el feedback, y entre el curso académico y las percepciones de evaluación. Esta información contribuye a un entendimiento más profundo del modo de aprender de los estudiantes en función de sus características y contexto. Palabras clave: estilos de aprendizaje, evaluación formativa, educación superior.
\end{abstract}

Abstract: Assessment contributes to students learning. Formative assessment is a useful strategy of the initial teacher training. Perceptions about the assessment of Chilean university Physical Education students ( $\mathrm{n}$ =95), depending on their learning style, gender and academic course were analyzed. The Learning Styles Questionnaire and the Spanish version of the Assessment Experience Questionnaire were used. The results showed negative perceptions about the assessment process. Significant differences were observed in the perceptions about the use of feedback depending on the learning style of the students $(F=1.062, p=.004)$. Significant differences were observed in the perception about the amount of effort $(F=$ 5.888, $p=.000)$, the suitability of the evaluation $(F=3.282, p=.015)$ and the shallow approach to the learning $(F=5.472, p=.001)$, depending on the academic course. Gender was not a determinant of perceptions. The findings suggest the use of traditional procedures of assessment (summative assessment) with the students. On the other hand, findings show the influence of learning stylesand the academic course on student perceptionsand learning styles and feedback. This information contributes to a deeper understanding of the learning mode of the students according to their characteristics and context.

Keywords:learning styles, formative assessment, higher education.

\section{Estilos de aprendizaje y evaluación formativa: Estudio con universitarios de Educación Física chilenos}

El cambio de concepción del aprendizaje desde el modelo tradicional de enseñanza hasta la actual formación por competencias, permite una perspectiva de mayor calidad pedagógica de los procesos educativos. Este aspecto se manifiesta en líneas de estudio sobre formación inicial docente, como el análisis de los estilos de aprendizaje (Gil-Madrona et al., 2007; González, Valenzuela, \& González, 2015), o la investigación en evaluación formativa (López Pastor, 2000; Monroy \& Hernández, 2014).

La profundización en los estilos de aprendizaje, por ejem-

Dirección para correspondencia [Correspodence address]: Dr. Jaime Serra Olivares. Facultad de Educación de Albacete. Universidad de CastillaLa Mancha. Plaza de la Universidad 3, 02071 (España). E-mail: Jaime. Serra@uclm.es plo, facilita la comprensión de los mecanismos mediante los cuales los estudiantes aprenden (Cordero et al., 2015; Izasa, 2014). Igualmente, el análisis de los procesos de evaluación formativa, proporciona información relevante sobre la importancia del feedback en la autoregulación del aprendizaje (Lopez Pastor et al., 2015).Así, ambas dimensiones han contribuido a la reflexión crítica sobre la formación universitaria, aunque curiosamente, nunca han sido relacionadas. A tal efecto, se percibe la necesidad de conocer las diferentes percepciones de los estudiantes respecto a su evaluación, en función de sus diferentes estilos de aprendizaje. Este conocimiento, permitirá un entendimiento superior de la utilización de la evaluación en los procesos de formación inicial docente en educación superior.

Nos referimos a los estilos de aprendizaje como aquellos 
esquemas de comportamiento flexibles y cambiantes que contribuyen a la construcción del conocimiento (Acevedo, Cavadla, \& Avis, 2015; Gil-Madrona, 2004; Gil-Madrona et al., 2007). Estos se ven influenciados, por ejemplo, por el contexto de aprendizaje, la experiencia, o las estrategias docentes (Bahamón et al., 2012; González et al., 2015).El modelo explicativo de Honey \& Mumdford el más aceptado, dado que permite discriminar diversas potencialidades en las cuatro dimensiones-estilos principales del aprendizaje-experiencia conocidos (Maureira, 2015). En la actualidad, se identifican hasta cuatro estilos de aprendizaje (activo, reflexivo, teórico, pragmático), y su combinación, que han demostrado diferencias en cuanto a sus características, potencial y debilidades (Acevedo et al., 2015; Bahamón et al., 2013; García \& De León, 2014; González et al., 2015; Villalba, 2015).

Se han analizado los estilos de aprendizaje en función de sus características (Camarero et al., 2000; Salas, 2014), el género, la edad, el curso de los participantes, o su relación con el rendimiento académico (Esguerra \& Guerrero, 2009). Los principales hallazgos muestran la presencia de un estilo más reflexivo en universitarios (Acevedo et al., 2015; González et al., 2015; Salas, 2014), principalmente en el género femenino (Bahamón et al., 2013; Blasco, et al., 2011; Villalba, 2015). Respecto a las diferencias en los estilos en función de la edad y el curso, los resultados indican una adaptación de la forma de aprender de los estudiantes, de un estilo más activo y/o teórico, a un estilo reflexivo, pragmático o combinado (e.g. Gil Madrona et al., 2007; Urval et al., 2014). De este modo, la sugerencia de la relación entre los estilos, las estrategias docentes, el contexto, y las características y habilidades del aprendiz para adaptarse.

Por último, el estudio de los estilos y el rendimiento académico sugiere una relación positiva entre el estilo reflexivo y el rendimiento académico (Esguerra \& Guerrero, 2009), aunque el estilo teórico también se ha mostrado determinante (Ossa \& Lagos, 2013).En cambio, los estilos activo y teórico han relacionado negativamente con el rendimiento académico(Camarero et al., 2000; Esguerro \& Guerrero, 2009), y con estrategias docentes propias de modelos tradicionales de enseńanza (Santizo et al., 2008). Igualmente, el estilo pragmático o la combinación de estilos, han demostrado una relación directa con aquellas metodologías más innovadoras (Bahamón et al., 2013; Cordero et al., 2015; Izasa, 2014). No obstante, cómo se mencionaba con anterioridad, los estilos de aprendizaje no han sido analizados en el contexto de metodologías innovadoras como la evaluación formativa, aspecto que limita el conocimiento sobre su posible relación.

La evaluación formativa se presenta como una estrategia eficaz dentro de la formación inicial docente (Hattie \& Timperley, 2007; López Pastor et al., 2013) y, sin embargo, no es utilizada con frecuencia (Brown, 2015; Margalef, 2014;
Monroy \& Hernández, 2014). Contribuye a la formación del aprendizaje, proporcionando información para facilitar el avance del estudiante (Brookhart, 2013). A pesar de ello, sigue existiendo el reto de complementar los sistemas de evaluación sumativa, centrada en exámenes finales, con aquellos más centrados en el aprendizaje y las necesidades del aprendiz (Blázquez, 1993; López Pastor \& Sicilia Camacho, 2015).

El estudio de los tipos de evaluación utilizada en educación superior ha contribuido a un entendimiento superior de los procesos de formación en las universidades. Existe una línea de estudio sobre el análisis del impacto de la evaluación sobre el aprendizaje de los estudiantes (Gibbs, 2002), que ha facilitado la identificación de aquellas condiciones que apoyan la evaluación formativa en el contexto educativo formal (Gibbs \& Simpson, 2003; Gibbs \& Simpson, 2004), e institucional (Gibbs, Simpson, \& Macdonald 2003).

Los resultados indican que existe disparidad en la forma de plantear la evaluación (utilización del feedback) en los diferentes programas, a pesar de la existencia de estándares internacionales (Gibbs \& Dunbar-Goddet, 2009). Se han analizado las diferencias en la percepción de los estudiantes sobre su evaluación en función del contexto universitario y las características de los programas. Los hallazgos sugieren la relación entre las características de los procesos evaluativos y la cantidad-calidad del feedback, y el sentido de las metas y los estándares académicos. No obstante, los estudiantes encuentran el feedback ineficaz cuando las demandas de la evaluación difieren entre los cursos, y cuando las formas de calificación son variadas (Jessop, El Hakim, \&Gibbs, 2014). De igual modo, se han observado diferentes patrones de evaluación y feedback en algunas modalidades como Humanidades, Educación Profesional y Ciencias. Se han apreciado diferencias en las demandas de la evaluación, la cantidad de feedback y la proporción de evaluaciones. Los resultados demuestran que, aunque no existen diferencias en las percepciones sobre la evaluación, algunos estudiantes como por ejemplo los de Humanidades, valoran positivamente la adecuación de su evaluación (Jessop \& Malekar, 2016). Por todo lo anterior, se percibe la necesidad de analizar el impacto de la evaluación sobre el aprendizaje, en función del perfil del aprendiz.

El propósito de este trabajo es, por tanto, analizar la percepción de la evaluación de estudiantes en un contexto de formación inicial docente en Educación Física en Chile. Igualmente, se pretende analizar las diferencias en las percepciones en función del estilo de aprendizaje, el género y curso académico. Las hipótesis de partida es que los estudiantes valorarán negativamente la experiencia en el proceso de evaluación, y se observarán diferentes percepciones en función de sus características (estilo, género y curso). Se prevé que aquellos estudiantes con estilos caracterizados por corrientes de enseńanza innovadoras (reflexivo, pragmático o combinado), presentarán percepciones más positivas que sus compañeros 
con otros estilos. Igualmente, se espera observar diferencias en las percepciones en función del género y el curso.

\section{Método}

\section{Participantes y Contexto}

Participaron los noventa y cinco estudiantes matriculados en Pedagogía en Educación Física de la Facultad de Educación de la Universidad Católica de Temuco $(n=95)$, en la región de la Araucanía, en Chile (69 chicos y 26 chicas, $22.8 \pm 0.3$ ańos de edad). El centro corresponde a una institución de educación superior privada, con financiación pública. Pertenece a la Agrupación de Universidades Regionales y está acreditada por la Comisión Nacional de Acreditación, al igual que la titulación. Cabe destacar que la región y la universidad se caracterizan por poseer altos índices de vulnerabilidad escolar, relacionados con problemas socioeconómicos y altas probabilidades de abandono escolar. Igualmente, se trata de la región con mayor diversidad cultural en el país, con el $31.2 \%$ de la población proveniente de pueblos indígenas, principalmente mapuche (JUNAEB-SINAE, 2015).

\section{Diseño}

Se realizó un estudio transversal y comparativo. Se encuestó a los participantes para conocer sus estilos de aprendizaje y percepción sobre la evaluación. Con este propósito, se utilizaron dos instrumentos, el Cuestionario de Estilos de Aprendizaje CHAEA-36 (Maureira, 2015), adaptado de la versión original deHoney\& Alonso (Alonso, 1991), y la versión en castellano del instrumentoAssessment Experience Questionnaire (AEQ) (Gibbs \& Dunbar-Goddet, 2007), traducida y validada por Nuńez \& Reyes (2014).

\section{Instrumentos}

El cuestionario CHAEA-36 ha sido utilizado en anteriores investigaciones sobre estilos de aprendizaje (Maureira, 2015; Serra-Olivares, Muñoz, Cejudo, \& Gil Madrona, 2017). Es una adaptación del instrumento CHAEA de Honey \& Alonso (Alonso, 1991), que consta de 36 ítems dicotómicos, en lugar de los 80 del cuestionario original. Se ha mostrado fiable y válido en contextos similares (Maueria, 2015). En todo caso, el análisis de la consistencia interna de los ítems con la muestra participante $(\alpha=<86)$, presenta su adecuación al estudio. El instrumento permite identificar el perfil y características del estudiante respecto al aprendizaje. Concretamente, clasifica la predominancia de un estilo (activo, reflexivo, teórico, pragmático o combinado), en función de las respuestas proporcionadas. En la tabla 1 se expone el procedimiento de identificación del estilo de aprendizaje del estudiante en función de la puntuación obtenida.

Tabla 1. Predominancia del estilo de aprendizaje del estudiante a partir de su puntuación en el Cuestionario de Estilos de Aprendizaje CHAEA-36 (Maureira, 2015).

\begin{tabular}{lllll}
\hline Nivel & Activo & Reflexivo & Teórico & Pragmático \\
\hline Bajo & $\leq 5$ & $\leq 6$ & $\leq 5$ & $\leq 5$ \\
Medio & $6-7$ & $7-8$ & $6-7$ & $6-7$ \\
Alto & $\geq 8$ & $\geq 9$ & $\geq 8$ & $\geq 8$ \\
\hline
\end{tabular}

Nota: Se codifica como Estilo de Aprendizaje Combinado cuando el estudiante ha obtenido puntuación Nivel Alto en más de un estilo, o en su defecto, sin poseer Nivel Alto en ningún estilo, presenta Nivel Medio en más de un estilo.

Por su parte, el AEQ valora la experiencia de evaluación del aprendizaje de los estudiantes. Se trata de un instrumento adaptado del original de Gibbs \& Simpson (2003). Incorpora nueve subcategorías, cinco del cuestionario original, dos del instrumento Course Experience Questionnaire (Ramsden,1991),dos sobre la percepción del estudiante respecto a la aproximación al estudio, y una sobre satisfacción con la enseńanza. Estas subcategorías proporcionan información específica sobre aspectos relevantes de la evaluación: cantidad de esfuerzo (ítems 6 y 13), cobertura del programa (ítems 4, 5, 11 y 17), cantidad y calidad de feedback (ítems3, 15 y 16), uso del feedback (ítems 1, 2 y 8), evaluación apropiada (ítems 10, 14 y18), claridad de los objetivos y criterios (ítems 7, 9 y 12), aproximación profunda(ítems 20, 21 y 22), aproximación superficial (ítems 19, 23 y 24), aprendizaje desde el examen (ítems 25, 26 y 27), y satisfacción con el proceso de enseñanza (ítem 28).

En total, contiene 28 ítems tipo Likert, 1 (totalmente en desacuerdo) 5 (totalmente de acuerdo), sobre la percepción del estudiante respecto a su proceso de evaluación. En este sentido, las puntuaciones altas en el cuestionario indican altos valores del constructo valorado por el instrumento, siguiendo como referencia instrumentos como la Encuesta $\mathrm{Na}$ cional del Estudiante, utilizada en el Reino Unido (región de aplicación del instrumento original) (Jessop et al., 2014). Así, el AEQ clasifica como buenos resultados aquellas subcategorías e ítems con puntuación "de acuerdo" o "fuertemente de acuerdo" (= y> 4) en la escala Likert, excepto la subcategoría "aprendizaje superficial", que clasifica como buenos resultados $(=0<2)$. El instrumento se ha mostrado fiable y válido en otros trabajos (Gibbs \& Dunbar-Goddet, 2007; Jessop et al., 2014), también en su traducción al castellano (Nuñez \& Reyes, 2014).En todo caso, se realizó el análisis factorial confirmatorio y se obtuvieron índices de bondad de ajuste adecuados $>.91$. Igualmente, el análisis de la consistencia interna de los ítems y factores con la muestra de estudio fue de $\alpha=<84$, justificando la utilización del instrumento para el 
análisis de los datos.

\section{Procedimiento y análisis de datos}

Los participantes dieron su consentimiento para la realización del estudio. Los cuestionarios fueron suministrados en cinco sesiones por el mismo investigador siguiendo el mismo protocolo, en la última semana del año académico 2015/16. Se organizó la información en función del curso en el que se encontraban los estudiantes $\left(1^{\circ}, 2^{\circ}, 3^{\circ}, 4^{\circ}\right.$ o $\left.5^{\circ} \mathrm{curso}\right)$, edad, género, y estilo de aprendizaje predominante, obtenido con el CHAEA-36(activo, reflexivo, teórico, pragmático, o combinado).

Se realizó el análisis de la homogeneidad de las varianzas de las variables analizadas. Este proceso cumplió con los supuestos necesarios para la utilización de pruebas paramétricas. Así, se desarrollaron sendos análisis (descriptivo e inferencial) para el tratamiento de los resultados. Con el propósito de analizar las percepciones de los estudiantes respecto a la evaluación, en función del género y el curso, se utilizó la prueba $A N O V A$ de un factor. Igualmente, se utilizó esta prueba para analizar las diferencias en las percepciones, en función del estilo de aprendizaje predominante. Por ejemplo, este procedimiento se realizó para analizar si estudiantes con determinados estilos de aprendizaje, poseían una percepción diferente sobre la adecuación del tipo de evaluación utilizada por los profesores. Finalmente, se calculó el tamaño del efecto en todos los análisis para comprobar la magnitud de las diferencias.

\section{Resultados}

\section{Predominancia de los estilos de aprendizaje}

El análisis muestra la predominancia del estilo combinado en el género masculino. Los chicos también poseen estilos activo y teórico en menor medida, y se aprecia un bajo porcentaje de estudiantes con estilos reflexivo y pragmático. El género femenino posee principalmente un estilo activo, seguido del combinado. Finalmente, se observa un porcentaje menor de chicas con un estilo teórico, y no existen estudiantes féminas con estilos reflexivo y pragmático (Figura 1).

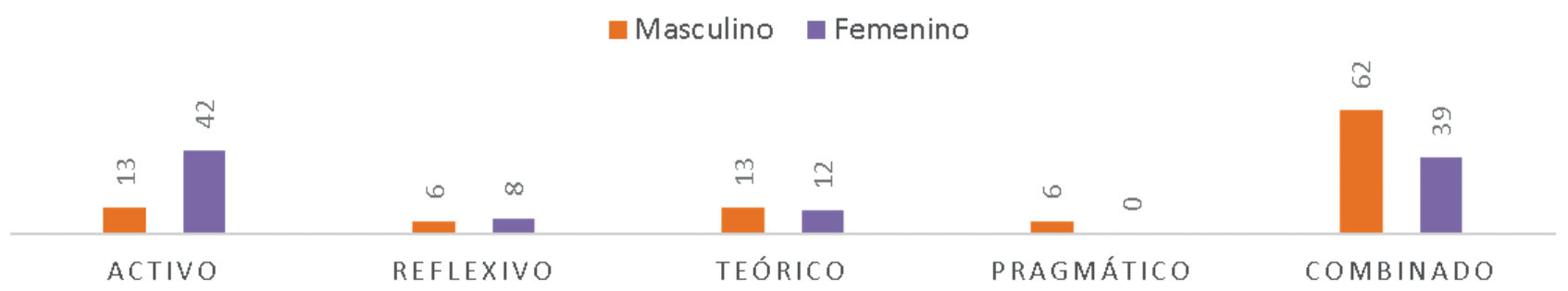

Figura 1. Predominancia del estilo de aprendizaje en función del género.

Respecto a las diferencias en los estilos en función del curso, $1^{\circ}$ posee estudiantes con diferentes estilos, aunque ningún estudiante posee el estilo pragmático. El $2^{\circ}$ curso posee un alto porcentaje de estudiantes con un estilo reflexivo, a diferencia de otros cursos. El $3^{\circ}$ curso posee, principalmente, estudiantes con estilos activo y pragmático. En el $4^{\circ}$ curso predominan los estudiantes con estilos reflexivo y pragmático. Finalmente, se observan en el $5^{\circ}$ curso estudiantes con estilos teórico o combinado, y algunos con un estilo activo, mientras que no existen estudiantes en este curso con un estilo pragmático (Figura 2).

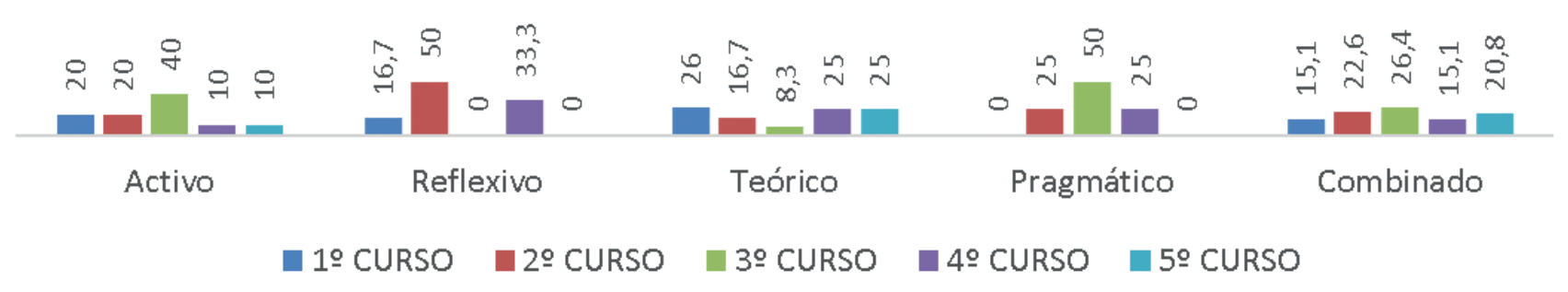

Figura 2. Predominancia del estilo de aprendizaje en función del curso académico. 
Percepciones sobre la evaluación en función del estilo de aprendizaje

El análisis descriptivo muestra percepciones similares de los estudiantes respecto a su evaluación (Tabla 2). No se observan diferencias significativas en su percepción en función del estilo de aprendizaje de los estudiantes en las subcategorías: cantidad de esfuerzo $(F=, 447, p=, 774)$, cobertura del programa $(F=, 112 ; p=, 978)$, cantidad y calidad de feedback $(F=$ $1,062 ; p=, 387)$, evaluación apropiada $(F=4,219 ; p=, 295)$, cla- ridad de los objetivos y criterios $(F=, 223 ; p=, 925)$, aproximación $\operatorname{profunda}(F=1,716 ; p=, 153)$, aproximación superficial $(F=, 547 ; p=, 702)$, aprendizaje desde el examen $(F=1,709 ; p$ $=, 155)$ y satisfacción con la enseñanza $(F=1,087 ; p=, 368)$. No obstante, las diferencias son significativas en la subcategoría uso del feedback $(F=1,062 ; p=, 004)$. La prueba posthoc de Bonferroni demuestra que los estudiantes con un estilo de aprendizaje activo presentan valores significativamente inferiores a sus compañeros con un estilo de aprendizaje teórico $(F=1,062 ; p=, 002, r=, 59)$.

Tabla 2. Percepciones de la evaluación en función del estilo de aprendizaje de los estudiantes.

\begin{tabular}{|c|c|c|c|c|c|c|c|c|c|c|}
\hline & \multicolumn{2}{|c|}{ Activo } & \multicolumn{2}{|c|}{ Reflexivo } & \multicolumn{2}{|c|}{ Teórico } & \multicolumn{2}{|c|}{ Pragmático } & \multicolumn{2}{|c|}{ Combinado } \\
\hline & M & DT & M & DT & M & DT & M & DT & M & DT \\
\hline Cantidad de esfuerzo & 3,48 & ,80 & 3,33 & 1,21 & 3,42 & ,93 & 2,88 & ,48 & 3,44 & ,84 \\
\hline Cobertura del programa & 3,24 &, 57 & 3,25 &, 45 & 3,19 &, 50 & 3,06 & ,31 & 3,25 & ,62 \\
\hline Cantidad y calidad de feedback & 2,68 & ,83 & 2,11 &, 72 & 2,22 & 1,08 & 2,08 &, 74 & 2,37 & ,78 \\
\hline Uso del feedback & 3,00 &, 80 & 3,94 &, 71 & 4,17 & ,73 & 3,58 &, 57 & 3,42 & ,89 \\
\hline Evaluación apropiada & 2,78 & ,91 & 2,17 & 96 & 2,19 & 1,20 & 2,08 & ,42 & 2,43 & ,83 \\
\hline Claridad de los objetivos y criterios & 3,40 & ,62 & 3,44 &, 34 & 3,36 & ,69 & 3,33 &, 47 & 3,49 &, 54 \\
\hline Aproximación profunda & 3,37 & ,83 & 3,28 & ,39 & 3,78 & ,72 & 2,75 &, 17 & 3,52 & ,76 \\
\hline Aproximación superficial & 3,03 & ,79 & 2,67 & 94 & 2,64 & 1,40 & 2,58 &, 32 & 2,87 & ,79 \\
\hline Aprendizaje desde el examen & 3,23 & ,82 & 3,72 & 1,39 & 4,08 & ,70 & 3,42 &, 57 & 3,58 & ,94 \\
\hline Satisfacción con la enseñanza & 4 & 1 & 4 & 1 & 4 & 1 & 4 & 1 & 4 & 1 \\
\hline
\end{tabular}

\section{Percepciones sobre la evaluación en función del género}

El análisis descriptivo demuestra puntuaciones ligeramente superiores del género masculino en algunas subcategorías del cuestionario (Tabla 3). Sin embargo, no se observan diferencias significativas en la percepción de los estudiantes respecto a su evaluación en función del género en las subcategorías: cantidad de esfuerzo $(F=, 048 ; p=, 828)$, cobertura del programa $(F=, 049 ; p=, 826)$, cantidad y calidad de feedback $(F=, 058 ; p=, 810)$, uso del feedback $(F=, 276 ; p=$ $, 601)$, evaluación apropiada $(F=, 301 ; p=, 585)$, aproximación $\operatorname{profunda}(F=, 262 ; p=, 610)$, aproximación superficial $(F=$ $1,395 ; p=, 241)$, aprendizaje desde el examen $(F=1,751 ; p=$ ,189) y satisfacción con la enseñanza $(F=, 091 ; p=, 764)$. Solamente se observa una tendencia hacia la significatividad en la subcategoría claridad de los objetivos y criterios $(F=3,711 ; p$ $=, 057, r=, 20)$, en la que el género masculino presenta una puntuación superior.
Tabla 3. Percepciones de la evaluación en función del género de los estudiantes.

\begin{tabular}{lcccc}
\hline & \multicolumn{2}{c}{ Masculino } & \multicolumn{2}{c}{ Femenino } \\
\cline { 2 - 5 } & $\underline{\mathrm{M}}$ & $\underline{\mathrm{DT}}$ & $\underline{\mathrm{M}}$ & $\underline{\mathrm{DT}}$ \\
\hline Cantidad de esfuerzo & 3,43 &, 85 & 3,38 &, 86 \\
\hline Cobertura del programa & 3,22 &, 56 & 3,25 &, 60 \\
\hline Cantidad y calidad de feedback & 2,38 &, 84 & 2,42 &, 82 \\
\hline Uso del feedback & 3,49 &, 84 & 3,38 & 1,04 \\
\hline Evaluación apropiada & 2,47 &, 88 & 2,36 &, 97 \\
\hline Claridad de los objetivos y criterios & 3,51 &, 53 & 3,27 &, 59 \\
\hline Aproximación profunda & 3,45 &, 78 & 3,54 &, 69 \\
\hline Aproximación superficial & 2,92 &, 84 & 2,68 &, 96 \\
\hline Aprendizaje desde el examen & 3,65 &, 94 & 3,37 &, 86 \\
\hline Satisfacción con la enseñanza & 4 & 1 & 4 & 1 \\
\hline
\end{tabular}

Percepciones sobre la evaluación en función del curso académico

El análisis descriptivo muestra una tendencia hacia valoraciones más negativas a medida que incrementa el curso. En este sentido, los estudiantes de $1^{\mathrm{o}}$ curso muestran valores supe- 
riores al resto, mientras que los de $4^{\circ}$ presentan valores muy bajos (Tabla 4). No se observan diferencias significativas en la percepción de los estudiantes respecto a su evaluación en función del curso en las subcategorías: uso del feedback ( $F=$ ,829; $p=, 571)$, claridad de los objetivos y criterios $(F=, 570 ; p=$ ,685), aproximación $\operatorname{profunda}(F=, 175 ; p=, 951)$, aprendizaje desde el examen $(F=1,580 ; p=, 187)$ y satisfacción con la enseñanza $(F=1,443 ; p=, 226)$.

Se observaron diferencias significativas en las subcategorías: cantidad de esfuerzo $(F=5,888 ; p=000)$, evaluación apropiada $(F=3,282 ; p=, 015)$ y aproximación superficial $(F=5,472 ; p=, 001)$. La prueba posthoc de Bonferroni mostró puntuaciones significativamente superiores del $1^{\circ}$ curso en la subcategoría cantidad de esfuerzo al compararlo con el $4^{\circ}$
$(F=3,282 ; p=, 013 ; r=, 47)$ y el $5^{\circ}(F=3,282 ; p=, 004 ; r=, 49)$, y puntuaciones significativamente superiores en la misma subcategoría del $2^{\circ}$ curso respecto al $4^{\circ}(F=3,282 ; p=, 021$, $r=, 47) \mathrm{y}$ el $5^{\circ}(F=3,282 ; p=, 006 ; r=, 50)$. El $1^{\circ}$ curso mostró también valores significantemente superiores al $4^{\circ}$ en la subcategoría evaluación apropiada $(F=3,282 ; p=, 015 ; r=, 49)$. El $1^{\circ}$ curso presentó valores significativamente superiores en la subcategoría aproximación superficial respecto a $3^{\circ}(F=$ $3,282 ; p=, 019 ; r=, 49), 4^{\circ}(F=3,282 ; p=, 000 ; r=, 61)$ y $5^{\circ}(F=$ $3,282 ; p=, 011 ; r=, 50)$ cursos. Por último, se observó una tendencia hacia la significatividad en las subcategorías cobertura del programa $(F=2,099 ; p=, 089)$ y cantidad y calidad de feedback $(F=2,231 ; p=, 072)$, en las que el $1^{\circ}$ curso mostró puntuaciones superiores a las de sus compañeros.

Tabla 4. Percepciones de la evaluación en función del curso de los estudiantes.

\begin{tabular}{lcccccccccc}
\hline & \multicolumn{3}{c}{ Primero } & \multicolumn{3}{c}{ Segundo } & \multicolumn{2}{c}{ Tercero } & \multicolumn{3}{c}{ Cuarto } & \multicolumn{2}{c}{ Quinto } \\
\cline { 2 - 11 } & M & DT & M & DT & M & DT & M & DT & M & DT \\
\hline Cantidad de esfuerzo & 3,87 &, 97 & 3,77 &, 80 & 3,44 &, 67 & 2,97 &, 69 & 2,88 &, 74 \\
Cobertura del programa & 3,47 &, 60 & 3,24 &, 55 & 3,33 &, 65 & 2,98 &, 34 & 3,06 &, 51 \\
Cantidad y calidad de feedback & 2,65 & 1,18 & 2,36 &, 83 & 2,60 &, 73 & 1,92 &, 55 & 2,31 &, 65 \\
Uso del feedback & 3,23 & 1,03 & 3,65 &, 91 & 3,39 &, 84 & 3,67 &, 71 & 3,35 &, 98 \\
Evaluación apropiada & 2,90 &, 96 & 2,58 &, 80 & 2,55 &, 73 & 1,90 &, 80 & 2,19 & 1,07 \\
Claridad de los objetivos y criterios & 3,60 &, 53 & 3,38 &, 49 & 3,49 &, 68 & 3,38 &, 59 & 3,38 &, 42 \\
Aproximación profunda & 3,44 & 1,09 & 3,53 &, 60 & 3,53 &, 62 & 3,35 &, 77 & 3,46 &, 81 \\
Aproximación superficial & 3,58 &, 68 & 2,97 &, 76 & 2,76 &, 76 & 2,33 &, 90 & 2,63 &, 93 \\
Aprendizaje desde el examen & 3,98 & 1,16 & 3,71 &, 84 & 3,33 &, 73 & 3,35 & 1,06 & 3,58 &, 85 \\
Satisfacción con la enseñanza & 4 & 1 & 4 & 1 & 4 & 1 & 4 & 1 & 4 & 1 \\
\hline
\end{tabular}

\section{Discusión}

El propósito de este trabajo fue analizar las percepciones sobre la evaluación de universitarios de Educación Física chilenos. Los hallazgos mostraron percepciones negativas, y se observarondiferencias en función del estilo de aprendizaje, género y el curso. A continuación, se discuten los resultados de acuerdo a el estado de la cuestión en la literatura científica.

\section{Percepciones sobre la evaluación}

Los estudiantes realizaron valoraciones negativas de su proceso evaluativo. Puntuaron por debajo de 4 en la escala Likert en todas las subcategorías del instrumento, y mostraron altos porcentajes de "aproximación superficial" al aprendizaje, como en otros estudios (Jessop \& Malekar, 2016).Estos resultados sugieren que los estudiantes podrían estar inmersos en un proceso de evaluación sumativa. Este proceso se caracteriza por la proporción de información y criterios detallados sobre los resultados, y la baja utilización del feedback(Jessop et al., 2014). Por el contrario, las valoraciones positivas (aproximación profunda, cobertura del programa, o aprendizaje desde el examen),están asociadas a procesos de evaluación formativa (Gibbs \& Dunbar-Goddet, 2009).

La utilización del feedback evaluativo por parte del estudiante se presenta como un elemento fundamental facilitador de aprendizajes (Hattie \& Timperley, 2007). Esta información puede ser utilizada para adaptar el proceso de aprendizaje, y contribuye a la construcción de estrategias de afrontamiento y toma de decisión (Gibbs, 2002). Estos aspectos deberían ser tenidos en cuenta por los profesionales de la educación superior, involucrados en los procesos de enseńanza-aprendizaje de estudiantes en etapas de formación inicial docente (Gibbs et al., 2003). El análisis de los procedimientos de evaluación utilizados, contribuirá a la caracterización de los escenarios que faciliten el establecimiento de procesos de evaluación formativa (Gibbs \& Simpson, 2003; Gibbs \& Simpson, 2004). 


\section{Percepciones sobre la evaluación y estilos de aprendizaje}

Los resultados indicaron la presencia de un estilo de aprendizaje combinado de los estudiantes, como en otros estudios (Bahamón et al., 2013; González et al., 2015), aunque se observó un alto porcentaje de chicas con un estilo activo. En este sentido, la presencia del estilo activo ha sido observada en estudiantes de Educación Física con anterioridad (Gil et al., 2007; Salas, 2014).La literatura sugiere que la combinación de estilos facilita la multimodalidad del aprendizaje, apoyando la construcción de conocimientos más complejos (Bahamón et al., 2013; Cordero et al., 2015; González et al., 2015). Por el contrario, el estilo activo y el teórico están más relacionados con procesos de enseñanza tradicional (Santizo et al., 2008).

Respecto a las percepciones sobre la evaluación, los estudiantes con un estilo pragmático mostraron valoraciones negativas en mayor medida que sus compañeros, aunque no se observaron diferencias significativas. El estilo pragmático ha sido observado en estudiantes inmersos en procesos innovadores de enseñanza (Izasa, 2014). Se observa en estudiantes que alcanzan aprendizajes profundos, y aplican de forma inmediata lo aprendido. Planifican y utilizan planes de acción eficientes, aunque tienen limitaciones cuando lo aprendido no tiene aplicación práctica contextualizada (Bahamón et al., 2013). Esto explicaría las valoraciones negativas de este tipo de estudiantes sobre la evaluación recibida, probablemente, de carácter sumativo.

Por otro lado, si bien las diferencias en las percepciones en función del estilo no fueron significativas en la mayoría de subcategorías, los estudiantes con un estilo teórico valoraron la utilización del feedback con puntuaciones significativamente superiores a sus compañeros con un estilo activo. Este hecho puede estar relacionado con las características del estilo teórico (Bahamón et al., 2013). Se observa en estudiantesdisciplinados, que razonan, y que se aproximan al nuevo aprendizaje de forma objetiva y estructurada (Acevedo et al., 2015; García \& De León, 2014; González et al., 2015; Villalba, 2015).

De manera que ciertas características del estilo teórico (ser disciplinado, o estructurado), podrían contribuir a una mejor utilización del feedback durante el proceso evaluativo. Cabe destacar que esta estrategia posee un gran potencial en la evaluación formativa (Magalef, 2014; Hattie \& Timperley, 2007). Este hallazgo es de gran importancia, dada la necesidad de conocer qué cualidades propias de cada estilo de aprendizaje, se relacionan más con estrategias de aprendizaje más eficientes.

\section{Percepciones sobre la evaluación y género}

Las percepciones del proceso evaluativo fueron similares, independientemente del género de los estudiantes. No obstan- te, las chicas valoraron con puntuaciones mucho más bajas la "claridad de los objetivos y criterios" de la evaluación. Estos resultados pueden estar relacionados con que las chicas presentaron un mayor predominio del estilo de aprendizaje activo. Este estilo se caracteriza por estudiantes creativos, innovadores y participativos, que se desencantan pronto y necesitan nuevos retos (Gil-Madrona et al., 2007). De esta manera, algunas cualidades del estilo activo, podrían favorecer la actitud crítica frente al proceso evaluativo. Este tipo de hallazgos permite un conocimiento más profundo de las formas de aprender de los estudiantes, tan relevante para la construcción de procesos de enseñanza de calidad (Hattie \& Timperley, 2007; López Pastor et al. 2013; López Pastor \& Sicilia Camacho, 2015).

Los resultados respecto a la predominancia del estilo de aprendizaje en función del género, difieren de otros en los que se apreció una mayor cantidad de chicas con un estilo reflexivo (Bahamón et al., 2013; Blasco, et al., 2011;Villalb, 2015), o una mayor cantidad de chicos con un estilo teórico(Gil-Madrona et al., 2007). Por este motivo, se percibe necesario seguir profundizando en las diferentes formas de aprender en función del género (Esguerra \& Guerrero, 2009; Acevedo et al., 2015), desarrollando análisis como los de este trabajo. Esta información permitirá conocer con mayor precisión cómo abordar factores tan importantes del proceso de enseńanza-aprendizaje, como la evaluación (Brookhart, 2013).

\section{Percepciones sobre la evaluación y curso académico}

El hecho de observar una tendencia hacia valoraciones más negativas de los últimos cursos, y puntuaciones significativamente más altas en las subcategorías cantidad de esfuerzo, evaluación apropiada y aproximación superficial (relacionadas con la evaluación sumativa) en el $1^{\circ}$, pudo ser debido a dos factores. El primer factor, que los estudiantes de $1^{\circ}$ estarían y habrían estado inmersos anteriormente en procesos de evaluación sumativa. Este hecho ha sido observado en estudios anteriores (Jessop et al., 2014). El segundo factor, que el estilo de aprendizaje habría influido directamente en las percepciones de los estudiantes.

Estudios anteriores han demostrado que los estudiantes modifican su estilo de aprendizaje durante el proceso educativo. Se adaptancon el tiempo, y adquieren un estilo combinado (Gil-Madrona et al., 2007;Bahamón et al., 2013; Villalba, 2015). En este sentido, es posible que los estudiantes de los últimos cursos de este trabajo, con un estilo más combinado (Serra-Olivares et al., 2017), percibiesen de forma más negativa el proceso evaluativo. Por este motivo, puede sugerirse que las cualidades de algunos estilos influyen en la forma de aprender e interactuar con el contexto de enseńanza. Este hallazgo es relevante para la construcción de procesos de ca- 
lidad (como el evaluativo), que se adecúen a las necesidades de los aprendices (Hattie \& Timperley, 2007; López Pastor et al. 2013).

Así, a pesar de encontrarse en un proceso de mejora respecto a los nuevos estándares de calidad de la Agencia Nacional de Acreditación (MINEDUC, 2015), se sugiere que la titulación de Educación Física de la institución participante, estaría utilizando procedimientos de evaluación más tradicional. Este aspecto, explicaría las percepciones negativas de los estudiantes de este trabajo sobre el proceso evaluativo, como en otros estudios (Brookhart, 2013; Brown, 2015). Por todo ello, son necesarias más investigaciones sobre los procedimientos evaluativos utilizados en etapas de formación inicial docente, y en los que se preste especial atención a las características de los estudiantes y sus estilos de aprendizaje, como en este trabajo. Esta información conducirá a la construcción de estrategias de enseńanza-aprendizaje eficientes, adaptadas a las particularidades del contexto (López Pastor \& Sicilia Camacho, 2015; Margalef, 2014; Monroy \& Hernández, 2014).

\section{Limitaciones del estudio}

Debido al tamaño de la muestra, los hallazgos de esta investigación deben ser examinados con cautela. Sería utópico concluir que el conocimiento de los estilos de aprendizaje es la clave para la implementación de procedimientos de evaluación innovadores y eficaces. No obstante, la información que aportan contribuye a un conocimiento más profundo de las formas de aprender a través del feedback evaluativo. Este factor es de gran relevancia para la implementación de procedimientos de evaluación formativa.
Por otro lado, si bien los instrumentos utilizados han permitido acceder a las diferentes percepciones de la evaluación en función de los estilos de aprendizaje, ha sido complicado ahondar en las diferencias observadas. Esto se debe a las características de los cuestionarios, con un diseño demasiado cuantitativo. En este sentido, sería interesante utilizar en futuros estudios instrumentos cualitativos como entrevistas, diarios, $\mathrm{u}$ otros instrumentos que permitan complementar la información obtenida. De este modo, se podrá lograr un mayor entendimiento de aquellas cualidades de los estilos que favorecen una mejor utilización del feedback evaluativo.

\section{Conclusiones}

Dados los hallazgos de este estudio, se intuye que los estudiantes observados podrían estar inmersos en procedimientos de evaluación tradicional. Los profesores deberían modificar, en este caso, sus procesos evaluativos, adecuándolos a las características de los alumnos y al nivel de los mismos. Para ello se sugiere modificar los feedbacks evaluativos a utilizar, orientándose a una evaluación centrada en las necesidades del alumnado. Con todo ello, son necesarios más estudios que analicen las características de los estudiantes y sus percepciones sobre el proceso evaluativo. Esta búsqueda permitirá un nivel de comprensión superior sobre las formas de aprender y planificación de la enseñanza, en etapas de formación inicial docente.

\section{Referencias}

1. Acevedo, D., Cavadla, S., \& Alvis, A. (2015). Estilos de Aprendizaje de los Estudiantes de la Facultad de Ingeniería de la Universidad de Cartagena (Colombia). Formación Universitaria, 8 (4), 15-22.

2. Alonso, C.M. (1991). Estilos de aprendizaje: Análisis y diagnóstico en estudiantes universitarios. Tesis doctoral. Facultad de Filosofía y Ciencias de La EducaciónUniversidad Complutense de Madrid.

3. Bahamón, M., Vianchá, M., Alarcón, L., \& Bohórquez, C. (2012). Estilos y estrategias de aprendizaje relacionados con el logro académico en estudiantes universitarios. Pensamiento Psicológico, 9 (1), 115-12.

4. Blasco, J.E., Romero, C., Mengual, S., Fernández-Revelles, A.B., Delgado, M.A. \& Vega, L. (2011). Estilo de aprendizaje de los estudiantes de magisterio de educación física y de ciencias del deporte de las universidades de Granada y Alicante. Cultura y educación, 23, 371-383.

5. Blázquez, D. (1993). Perspectivas de la evaluación en Educación Física y Deporte. Apunts, 31, 5-16.

6. Brown, S. 2015. Learning, Teaching and Assessment in Higher Education: Global Perspectives. London: Palgrave-Macmillan.

7. Brookhart, S. M. 2013. How to Create and Use Rubrics for Formative Assessment and Grading. Alexandria, VA: Association for Supervision and Curriculum Development.

8. Camarero, F., Buey, F., \& Herrero, J. (2000). Estilos y estrategias de aprendizaje en estudiantes universitarios. Psicothema, 12(4), 615-622.

9. Cordero, E., Lizano, C., Ortiz, A., \& Arias, F. (2015). Relationship between learning style and academic performance in students of the pharmacy program at Universidad de Costa Rica. Revista Digital de Investigación en Docencia Universitaria, $O$ (2), 49-63.

10. Dunbar-Goddet, H., \& G. Gibbs. in press. A research tool for evaluating the effects of programme assessment environments on student learning: The Assessment Experience Questionnaire (AEQ). Assessment \& Evaluation in Higher Education.

11. Esguerrra, G., \& Guerrero, P. (2009). Estilos de aprendizaje y rendimiento académico en estudiantes de Psicología. Perspectivas en Psicologí, 6(1), 97-109.

12. García, G., \& De León, B. (2014). Estilos de Aprendizaje. Una revisión. Revista electrónica de la divulgación de la investigación, 6, 1-8.

13. Gibbs, G.,\& Dunbar-Goddet, H. (2009) Characterising programmelevelassessment environments that support learning, Assessment \& Evaluation in Higher Education, 34:4, 481-489.

14. Gibbs, G. (2002). Evaluation of the impact of formative assessment on student learning behaviour. European Association for Research into Learning and Instruction. Newcastle: Northumbria University.

15. Gibbs, G., \& Simpson, C. (2003). Measuring the response of students to assessment: The Assessment Experience Questionnaire. Comunicación presentada en el 11th International Improving Students Learning Symposium. Hinckley.

16. Gibbs, G., \& Simpson, C.(2004). Conditions under which assessment supports student learning. Learning and Teaching in Higher Education $1: 3-31$. 
17. Gibbs, G., C. Simpson, \&R. Macdonald. 2003. Improving student learning through changing assessment-A conceptual and practical framework. European Association for Research into Learning and Instruction Conference, Padova, Italy.

18. Gil Madrona, P. (2004). Estilos de aprendizaje y Educación Física. En Alonso, C. \& Gallego, D. (Eds.) I Congreso Internacional de Estilos de Aprendizaje. Madrid: UNED

19. Gil Madrona, P., Contreras, O. P., Isabel, G., González, S., García, L., De Moya, M. d., \& López, A. (2007). Estilos de aprendizaje de los estudiantes de magisterio: Especial. Profesorado, Revista de Currículum y formación de profesorado, 12(2), 1-19.

20. González, E., Valenzuela, G., \& González, A. (2015). Diferencias significativas de los estilos de aprendizaje con las características del estudiante universitario en México. Revista de Estilos de Aprendizaje, 8 (15), 201-221.

21. Hattie, J., \& H. Timperley. (2007). The Power of Feedback. Review of Educational Research 77(1), 81-112.

22. Junta Nacional de Auxilio Escolar y Becas, JUNAEB (2015). SINAE: Sistema Nacional de Asignación con equidad para becas JUNAEB. Santiago, Chile: JUNAEB, Dirección General.

23. Jessop, T., El Hakim, Y.,\& Gibbs, G. (2014).The whole is greaterthan the sum of its parts: a large-scale study of students' learning in response to different programme assessment patterns.Assessment \& Evaluation in Higher Education, 39(1), 73-88.

24. Jessop, T., \& Maleckar, B. (2016). The influence of disciplinary assessment patterns on student learning: a comparative study.Studies in Higher Education, 41(4), 696-711.

25. López Pastor, V. M., Pintor, P., Muros, B., \& Webb, G.. (2013) Formative Assessment Strategies and Their Effect on Student Performance and on Student and Tutor Workload: The Results of Research Projects Undertaken in Preparation for Greater Convergence of Universities in Spain within the European Higher Education Area (EHEA). Journal of Further and Higher Education, 37 (2): 163-180.

26. López Pastor, V. (2000). Buscando una evaluación formativa en educación física: Análisis crítico de la realidad existente, presentación de una propuesta y análisis general de su puesta en práctica. Apunts, 4(62), $15-26$
27. López Pastor, V., \& Sicilia-Camacho, A. (2015). Formative and shared assessment in higher education. Lessons learned and challenges for the future, Assessment \& Evaluation in Higher Education

28. Margalef, G. (2014). Evaluación formativa de los aprendizajes en el contexto universitario: Resistencias y paradojas del profesorado. Educación $X X 1,17(2), 1-12$

29. Maureira, F. (2015). CHAEA-36: Adaptación del cuestionario HoneyAlonso de estilos de aprendizajes para estudiantes de educación física de Chile. Revista Electrónica de Psicología Iztacala, 18(3), 1133-1152.

30. Monroy, F., \& Hernandez, F. (2014). Factores que influyen en los enfoques de aprendizaje universitario. Una revisión sistemática. Revista Educación XXI, 17 (2), 105-124.

31. Nuñez, J., \& Reyes, C. (2014). La evaluación del aprendizaje de estudiantes: validación espańola del Assessment Experience Questionnaire (AEQ). Estudios Sobre Educacion, 26, 63-77.

32. Ossa, C., \& Lagos, N. (2013). Estilos de Aprendizaje y rendimiento académico en estudiantes de Pedagogía de Educación General Básica (Primaria de una universidad pública en Chile). Revista Estilos de Aprendizaje, 6(11), 178-189.

33. Ramsden, P. (1991). A performance indicator of teaching quality in higher education: The Course Experience Questionnaire. Studies in Higher Education, 16,129-150.

34. Salas, J. (2014). Estilos de aprendizaje en estudiantes de la Escuela de Ciencias del Movimiento Humano y Calidad de Vida, Universidad Nacional, Costa Rica. Revista electronica EDUCARE, 23(3), 159-171.

35. Santizo, J., García, L., \& Gallego, D. (2008). Dos métodos para la identificación de diferencias de estilos de aprendizaje entre estudios donde se ha aplicado el CHAEA. Revista Estilos de Aprendizaje, 1(1), $28-42$.

36. Urval, R., Kamath, A., Ullal, S., Shenoy, A., Shenoy, N., \& Udupa, L. (2014). Assessment of learning styles of undergraduate medical students using the VARK questionnaire and the influence of sex and academic performance.Advances in Physiology Education, 38(3), 216-220.

37. Villalba, A. (2015). Estilos de aprendizaje en alumnos universitarios de profesorado en Biología y licenciatura en Biodiversidad. Revista Estilos de Aprendizaje, 8(16), 79-100. 